\title{
Ulam Stability of $n$-th Order Delay Integro-Differential Equations
}

\author{
Shuyi Wang * and Fanwei Meng
}

check for

updates

Citation: Wang, S.; Meng, F. Ulam Stability of an $n$-th Order Delay Integro-Differential Equations. Mathematics 2021, 9, 3029. https:/ / doi.org/10.3390/math9233029

Academic Editor: Alberto Cabada

Received: 20 October 2021

Accepted: 19 November 2021

Published: 26 November 2021

Publisher's Note: MDPI stays neutral with regard to jurisdictional claims in published maps and institutional affiliations.

Copyright: (c) 2021 by the authors. Licensee MDPI, Basel, Switzerland. This article is an open access article distributed under the terms and conditions of the Creative Commons Attribution (CC BY) license (https:/ / creativecommons.org/licenses/by/ $4.0 /)$.
School of Mathematical Sciences, Qufu Normal University, Qufu 273165, China; fwmeng@qfnu.edu.cn

* Correspondence: sywang_math@126.com

\begin{abstract}
In this paper, the Ulam stability of an $n$-th order delay integro-differential equation is given. Firstly, the existence and uniqueness theorem of a solution for the delay integro-differential equation is obtained using a Lipschitz condition and the Banach contraction principle. Then, the expression of the solution for delay integro-differential equation is derived by mathematical induction. On this basis, we obtain the Ulam stability of the delay integro-differential equation via Gronwall-Bellman inequality. Finally, two examples of delay integro-differential equations are given to explain our main results.
\end{abstract}

Keywords: Ulam stability; delay integro-differential equation; Gronwall-Bellman inequality

\section{Introduction}

In the year 1940, Ulam [1] put forward an abstract problem: under what conditions is the exact solution of an equation closed to the approximate solution? In the year 1941, in order to solve the problem raised by Ulam, Hyers [2] studied the functional equation in Banach space and gave the definition of Hyers-Ulam stability. In the year 1978, based on the work of Hyers, Rassias [3] gave the definition of Hyers-Ulam-Rassias stability. These two kinds of stability are called Ulam stability. After that, scholars began to study the Ulam stability of some solvable equations. See [4-7] and the references therein. Recently, the Ulam stability of delay differential equations and delay integro-differential equations has been discussed. See [8-13]. There are many results about the Ulam stability of delay differential equations. However, there are a few results about the Ulam stability of delay integro-differential equations.

In fact, delay integro-differential equations are usually used to describe many natural phenomena in the fields of thermodynamics, mechanics, mechanical engineering and control. See [14-17]. Mechanical processes, such as rigid heat conduction process [18] and the motion of charged particles with a delayed interaction [19], can be modeled by delay integro-differential equations. Furthermore, the delay integro-differential equation is an appropriate model for studying the effect of tire dynamics on a vehicle shimmy [20] and the optimal control of a size-structured population [21], which is one of its important applications. The mathematical model related to the delay integro-differential equation is an interesting memory effect model. However, new difficulties may arise when delay and integro-differential equations are introduced simultaneously. Some topics about delay integro-differential equations, such as the existence and uniqueness of solutions and Ulam stability, have attracted the attention of many scholars. See [12,14,15,22].

In [23], Otrocol studied Ulam stability of a first-order delay differential equation:

$$
u^{\prime}(t)=g(t, u(t), u(\tau(t))), t \in\left[t_{0}, t_{1}\right]
$$

where $g \in C\left(\left[t_{0}, t_{1}\right] \times \mathbb{R}^{2}, \mathbb{R}\right)$; delay function $\tau(t) \leq t, \tau \in C\left(\left[t_{0}, t_{1}\right],\left[t_{0}-l, t_{1}\right]\right), l>0$. 
In 2015, Kendre [24] discussed the existence of a solution for an integro-differential equation:

$$
\left\{\begin{array}{l}
u^{\prime}(t)=g\left(t, u(u(t)), \int_{t_{0}}^{t} k(t, s) u(u(s)) d s\right), t \in\left[t_{0}, t_{1}\right], \\
u\left(t_{0}\right)=u_{0}
\end{array}\right.
$$

where $G=\left[t_{0}, t_{1}\right], g \in C\left(G^{3}, G\right) ; k \in C\left(G^{2}, G\right) ; t_{0}, u_{0} \in G$.

In 2016, Sevgin [25] investigated the Ulam stability of the Volterra integro-differential equation:

$$
u^{\prime}(t)=f(t, u(t))+\int_{0}^{t} g(t, s, u(s)) d s, t \in\left[0, t_{0}\right],
$$

where $f \in C\left(\left[0, t_{0}\right] \times \mathbb{R}, \mathbb{R}\right) ; g \in C\left(\left[0, t_{0}\right] \times\left[0, t_{0}\right] \times \mathbb{R}, \mathbb{R}\right)$.

In 2018, Kishor [26] established the Ulam stability of the semilinear Volterra integrodifferential equation:

$$
u^{\prime}(t)=T u(t)+g\left(t, u(t), \int_{0}^{t} z(t, s, u(s)) d s\right), t \in\left[0, t_{0}\right]
$$

where $T: U \rightarrow U$ is the infinitesimal generator; $U$ is Banach space; $g \in C\left(\left[0, t_{0}\right] \times \mathbb{R}^{2}, \mathbb{R}\right)$; $z \in C\left(\left[0, t_{0}\right] \times \mathbb{R}^{2}, \mathbb{R}\right)$.

In 2019, Zada [27] obtained the Ulam stability for the following $n$-th order delay differential equation:

$$
\left\{\begin{array}{l}
u^{(n)}(s)=z\left(s,\left\{u^{(0)}\right\},\left\{u^{(1)}\right\}, \ldots,\left\{u^{(n-1)}\right\}\right), s \in\left[s_{0}, s_{0}+\eta\right] \\
u(s)=\chi(s), s \in\left[s_{0}-\zeta, s_{0}\right]
\end{array}\right.
$$

where $\left\{u^{(j)}\right\}=\left\{u^{(j)}(s), u^{(j)}\left(\lambda_{1}(s)\right), u^{(j)}\left(\lambda_{2}(s)\right), \ldots, u^{(j)}\left(\lambda_{k}(s)\right)\right\}, j=0,1, \ldots, n-1$; delay functions $\lambda_{k} \in C\left(\left[s_{0}, s_{0}+\eta\right],\left[s_{0}-\zeta, s_{0}+\eta\right]\right), \lambda_{k}(s) \leq s, k \in \mathbb{Z}_{+} ; \zeta>0, \eta>0, s_{0}$ are constants; $z \in C(\tilde{B}, \mathbb{R}), \tilde{B} \subset\left[s_{0}, s_{0}+\eta\right] \times \mathbb{R}^{n(k+1)}$ is closed set; $\chi:\left[s_{0}-\zeta, s_{0}\right] \rightarrow \mathbb{R}$.

However, the existence and uniqueness of solutions and Ulam stability for $n$-th delay integro-differential equations have not been studied hitherto. Inspired by $[24,25,27]$, we study Ulam stability for the following $n$-th order delay integro-differential equation:

$$
\left\{\begin{array}{l}
u^{(n)}(s)=z\left(s, u^{(0)}, \ldots, u^{(n-1)}, u^{(0)}(\lambda), \ldots, u^{(n-1)}(\lambda), \int_{s_{0}}^{s} g\left(\tau, u^{(0)}, \ldots, u^{(n-1)}\right) d \tau\right), \\
u(s)=\chi(s), s \in\left[s_{0}-\zeta, s_{0}\right] \\
u^{(j)}\left(s_{0}\right)=\chi^{(j)}\left(s_{0}\right), j=1, \ldots, n-1
\end{array}\right.
$$

where the definition domain of the first formula of Equation (3) is $\left[s_{0}, s_{0}+\eta\right]$, where $u^{(j)}=$ $u^{(j)}(s), u^{(j)}(\lambda)=u^{(j)}(\lambda(s)), j=0, \ldots, n-1$; delay function $\lambda(s) \leq s, \lambda \in C\left(\left[s_{0}, s_{0}+\eta\right]\right.$, $\left.\left[s_{0}-\zeta, s_{0}+\eta\right]\right) ; z \in C(B, \mathbb{R}), B \subset\left[s_{0}, s_{0}+\eta\right] \times \mathbb{R}^{2 n+1}$ is closed set; $g \in C(H, \mathbb{R}), H \subset$ $\left[s_{0}, s_{0}+\eta\right] \times \mathbb{R}^{n}$ is closed set; $\chi \in C^{n}\left(\left[s_{0}-\zeta, s_{0}\right], \mathbb{R}\right)$.

The aim of our paper is to study the Ulam stability and the existence and uniqueness of solutions for Equation (3). The main tools used in this paper are Lipschitz conditions and Gronwall-Bellman inequality.

The remainder of the paper is organized as follows: In Section 2, we give definitions and lemmas, which are essential for Section 3. In Section 3, we state some Lipschitz conditions, which will be helpful to prove the existence and uniqueness results for a delay integro-differential equation; then the Ulam stability for the delay integro-differential equation is given. In Section 4, we give two examples to illustrate main results. 


\section{Preliminaries}

In this paper, we denote $\mathbb{R}^{+}:=[0, \infty), J_{1}:=\left[s_{0}-\zeta, s_{0}+\eta\right], J_{2}:=\left[s_{0}, s_{0}+\eta\right], J_{3}:=$ $\left[s_{0}-\zeta, s_{0}\right]$. Let $C\left(J_{1}, \mathbb{R}\right)$ be real Banach space of all continuous functions with norm:

$$
\|u\|=\sup \left\{|u(s)| ; s \in J_{1}\right\} .
$$

Definition 1. Equation (3) is Hyers-Ulam stable on $J_{1}$ if there exists $C>0$ such that for $\theta>0$ and each solution $v(s)$ of the inequality

$$
\left\{\begin{array}{l}
\left|v^{(n)}(s)-z\left(s, v^{(0)}, \ldots, v^{(n-1)}(\lambda), \int_{s_{0}}^{s} g\left(\tau, v^{(0)}, \ldots, v^{(n-1)}\right) d \tau\right)\right| \leq \theta, s \in J_{2}, \\
|v(s)-\chi(s)| \leq \theta, s \in J_{3}
\end{array}\right.
$$

there exists a solution $u(s) \in C\left(J_{1}, \mathbb{R}\right) \cap C^{n}\left(J_{2}, \mathbb{R}\right)$ of Equation (3) with

$$
|v(s)-u(s)| \leq C \cdot \theta, s \in J_{1} .
$$

Definition 2. Equation (3) is Hyers-Ulam-Rassias stable with respect to $\sigma(s)$ on $J_{1}$ if there exists $K_{z, g, \sigma}>0$ such that for each solution $v(s)$ of the inequality

$$
\left\{\begin{array}{l}
\left|v^{(n)}(s)-z\left(s, v^{(0)}, \ldots, v^{(n-1)}(\lambda), \int_{s_{0}}^{s} g\left(\tau, v^{(0)}, \ldots, v^{(n-1)}\right) d \tau\right)\right| \leq \sigma(s), s \in J_{2}, \\
|v(s)-\chi(s)| \leq \sigma(s), s \in J_{3}
\end{array}\right.
$$

there exists a solution $u(s) \in C\left(J_{1}, \mathbb{R}\right) \cap C^{n}\left(J_{2}, \mathbb{R}\right)$ of Equation (3) with

$$
|v(s)-u(s)| \leq K_{z, g, \sigma} \cdot \sigma(s), s \in J_{1} .
$$

Lemma 1 (see [28]). Assume that $f \in C(\mathbb{R}, Q)$, then $n$-th repeated integrable of $f$ based at $s_{0}$,

$$
f^{(-n)}(s)=\int_{s_{0}}^{s} \int_{s_{0}}^{s_{1}} \int_{s_{0}}^{s_{2}} \int_{s_{0}}^{s_{3}} \ldots \int_{s_{0}}^{s_{n-1}} f\left(s_{n}\right) d s_{n} d s_{n-1} \ldots d s_{2} d s_{1}
$$

is given by

$$
f^{(-n)}(s)=\frac{1}{(n-1) !} \int_{s_{0}}^{s}(s-\tau)^{n-1} f(\tau) d \tau .
$$

Theorem 1. A function $u(s) \in C\left(J_{1}, \mathbb{R}\right) \cap C^{n}\left(J_{2}, \mathbb{R}\right)$ is a solution of the delay integro-differential equation

$$
\left\{\begin{array}{l}
u^{(n)}(s)=z\left(s, u^{(0)}, \ldots, u^{(0)}(\lambda), \ldots, u^{(n-1)}(\lambda), \int_{s_{0}}^{s} g\left(\tau, u^{(0)}, \ldots, u^{(n-1)}\right) d \tau\right), s \in J_{2}, \\
u(s)=\chi(s), s \in J_{3}, \\
u^{(j)}\left(s_{0}\right)=\chi^{(j)}\left(s_{0}\right), j=1, \ldots, n-1,
\end{array}\right.
$$

if and only if $u(s)$ is a solution of the integral equation

$$
u(s)=\left\{\begin{array}{l}
\sum_{j=0}^{n-1} \frac{\left(s-s_{0}\right)^{j} \chi^{(j)}\left(s_{0}\right)}{j !}+\frac{1}{(n-1) !} \int_{s_{0}}^{s}(s-\tau)^{n-1} \\
\cdot z\left(\tau, u^{(0)}, \ldots, u^{(0)}(\lambda), \ldots, u^{(n-1)}(\lambda), \int_{s_{0}}^{\tau} g\left(r, u^{(0)}, \ldots, u^{(n-1)}\right) d r\right) d \tau, s \in J_{2}, \\
\chi(s), s \in J_{3} .
\end{array}\right.
$$


Proof. For $n=1$, from (6), we have

$$
\begin{aligned}
& u^{(1)}(s)=z\left(s, u^{(0)}, u^{(0)}(\lambda), \int_{s_{0}}^{s} g\left(\tau, u^{(0)}\right) d \tau\right), \\
& u\left(s_{0}\right)=\chi\left(s_{0}\right),
\end{aligned}
$$

then by integral formula, we have

$$
u(s)=\chi\left(s_{0}\right)+\int_{s_{0}}^{s} z\left(\tau, u^{(0)}, u^{(0)}(\lambda), \int_{s_{0}}^{\tau} g\left(r, u^{(0)}\right) d r\right) d \tau, s \in J_{2} .
$$

This means that (7) holds for $n=1$.

For $n=k$, from (6), we have

$$
\begin{aligned}
& u^{(k)}(s)=z\left(s, u^{(0)}, \ldots, u^{(0)}(\lambda), \ldots, u^{(k-1)}(\lambda), \int_{s_{0}}^{s} g\left(\tau, u^{(0)}, \ldots, u^{(k-1)}\right) d \tau\right), \\
& u^{(j)}\left(s_{0}\right)=\chi^{(j)}\left(s_{0}\right), j=1, \ldots, k-1 .
\end{aligned}
$$

Assume for $n=k,(7)$ holds; that is,

$$
\begin{aligned}
u(s)= & \sum_{j=0}^{k-1} \frac{\left(s-s_{0}\right)^{j} \chi^{(j)}\left(s_{0}\right)}{j !}+\frac{1}{(k-1) !} \int_{s_{0}}^{s}(s-\tau)^{k-1} \cdot z\left(\tau, u^{(0)}, \ldots, u^{(0)}(\lambda), \ldots,\right. \\
& \left.u^{(k-1)}(\lambda), \int_{s_{0}}^{\tau} g\left(r, u^{(0)}, \ldots, u^{(k-1)}\right) d r\right) d \tau, s \in J_{2} .
\end{aligned}
$$

Hence, for $n=k+1$, from (6), we have

$$
u^{(k+1)}(s)=z\left(s, u^{(0)}, \ldots, u^{(0)}(\lambda), \ldots, u^{(k)}(\lambda), \int_{s_{0}}^{s} g\left(\tau, u^{(0)}, \ldots, u^{(k)}\right) d \tau\right),
$$

then by the integral formula, we have

$$
u^{(k)}(s)=\chi^{(j)}\left(s_{0}\right)+\int_{s_{0}}^{s} z\left(\tau, u^{(0)}, \ldots, u^{(0)}(\lambda), \ldots, u^{(k)}(\lambda), \int_{s_{0}}^{\tau} g\left(r, u^{(0)}, \ldots, u^{(k)}\right) d r\right) d \tau .
$$

From the inductive hypothesis and Lemma 1, we have

$$
\begin{aligned}
u(s)= & \sum_{j=0}^{k-1} \frac{\left(s-s_{0}\right)^{j} \chi^{(j)}\left(s_{0}\right)}{j !}+\frac{1}{(k-1) !} \int_{s_{0}}^{s}(s-\tau)^{k-1} \cdot \chi^{(j)}\left(s_{0}\right) d \tau \\
& +\frac{1}{(k-1) !} \int_{s_{0}}^{s}(s-\tau)^{k-1} \int_{s_{0}}^{\tau} z\left(r, u^{(0)}, \ldots, u^{(0)}(\lambda), \ldots, u^{(k)}(\lambda),\right. \\
& \left.\int_{s_{0}}^{r} g\left(t, u^{(0)}, \ldots, u^{(k)}\right) d t\right) d r d \tau \\
= & \sum_{j=0}^{k} \frac{\left(s-s_{0}\right)^{j} \chi^{(j)}\left(s_{0}\right)}{j !}+\frac{1}{k !} \int_{s_{0}}^{s}(s-\tau)^{k} \cdot z\left(\tau, u^{(0)}, \ldots, u^{(0)}(\lambda), \ldots,\right. \\
& \left.u^{(k)}(\lambda), \int_{s_{0}}^{\tau} g\left(r, u^{(0)}, \ldots, u^{(k)}\right) d r\right) d \tau .
\end{aligned}
$$

Hence, by mathematical induction, the conclusion is estabilished.

Lemma 2 (see [23]). (abstract Gronwall lemma) Let $(Y, d)$ be an ordered metric space and $A: Y \longrightarrow Y$ be an increasing Picard operator $\left(F_{A}=\left\{x_{A}{ }^{*}\right\}\right)$. Then, for $x \in Y, x \leq A(x)$ implies $x \leq x_{A}{ }^{*}$ and $x \geq A(x)$ implies $x \geq x_{A}{ }^{*}$. 
Lemma 3 (see [29]). (Gronwall lemma) Assume that $u(s), b(s) \in C\left([a,+\infty), \mathbb{R}^{+}\right), T>0$ is constant. If $u(s) \in C\left([a,+\infty), \mathbb{R}^{+}\right)$satisfies

$$
u(s) \leq T+\int_{a}^{s} b(\tau) u(\tau) d \tau, s \in[a,+\infty),
$$

then

$$
u(s) \leq T \exp \left(\int_{a}^{s} b(\tau) d \tau\right), s \in[a,+\infty)
$$

Lemma 4 (see [30]). Assume that $w(s), d(s), l(s), m(s), n(s) \in C\left(\mathbb{R}^{+}, \mathbb{R}^{+}\right)$and $d(s), l(s)$ are nondecreasing functions on $\mathbb{R}^{+}$. If $w(s)$ satisfies the delay integral inequality

$$
w^{h}(s) \leq d(s)+l(s) \int_{0}^{s}\left[m(\tau) w^{a}(\sigma(\tau))+n(\tau) w^{b}(\tau)+\int_{0}^{\tau} z(r) w^{c}(r) d r\right] d \tau, s \in \mathbb{R}^{+},
$$

with initial conditions

$$
w(s)=r(s), s \in[\alpha, 0] ; r(\sigma(s)) \leq d(s)^{\frac{1}{h}}, s \in \mathbb{R}^{+} \text {and } \sigma(s) \leq 0,
$$

where $h \neq 0, h \geq a \geq 0, h \geq b \geq 0, h \geq c \geq 0$ and $h, a, b, c$ are constants; $\sigma(s) \in C\left(\mathbb{R}^{+}, \mathbb{R}\right)$ and $\sigma(s) \leq s ;-\infty<\alpha=\inf \left\{\sigma(s), s \in \mathbb{R}^{+}\right\} \leq 0$ and $r(s) \in C\left([\alpha, 0], \mathbb{R}^{+}\right)$, then

$$
w(s) \leq\left[d(s)+l(s) U(s) \exp \left(\int_{0}^{s} V(\tau) d \tau\right)\right]^{\frac{1}{h}}
$$

where, for any $H>0, s \in \mathbb{R}^{+}$,

$$
\begin{aligned}
U(s) & =\int_{0}^{s} m(\tau)\left[\left(\frac{a}{h} H^{\frac{a-h}{h}} d(\tau)+\frac{h-a}{h} H^{\frac{a}{h}}\right)+g(\tau, s)\left(\frac{b}{h} H^{\frac{b-h}{h}} d(\tau)+\frac{h-b}{h} H^{\frac{b}{h}}\right)\right. \\
& \left.+\int_{0}^{\tau} z(r)\left(\frac{c}{h} H^{\frac{c-h}{h}} d(r)+\frac{h-c}{h} H^{\frac{c}{h}}\right) d r\right] d \tau, \\
V(s) & =\left(\frac{a}{h} H^{\frac{a-h}{h}} m(s)+\frac{b}{h} H^{\frac{b-h}{h}} n(s)\right) l(s)+\int_{0}^{s} z(\tau) l(\tau) \frac{c}{h} H^{\frac{c-h}{h}} d \tau .
\end{aligned}
$$

\section{Existence and Stability Results for the Delay Integro-Differential Equation}

Before stating the main theorems, we give the following Lipschitz conditions:

$$
\begin{aligned}
& \left|z\left(s, u^{(0)}, \ldots, u^{(n-1)}, \bar{u}^{(0)}, \ldots, \bar{u}^{(n-1)}, \tilde{u}\right)-z\left(s, v^{(0)}, \ldots, v^{(n-1)}, \bar{v}^{(0)}, \ldots, \bar{v}^{(n-1)}, \tilde{v}\right)\right| \\
& \leq K_{z} \sum_{j=0}^{n-1}\left[\left|u^{(j)}-v^{(j)}\right|+\left|\bar{u}^{(j)}-\bar{v}^{(j)}\right|\right]+M|\tilde{u}-\tilde{v}|
\end{aligned}
$$

where $K_{z}>0, M>0, \bar{u}^{(j)}=u^{(j)}(\lambda), \bar{v}^{(j)}=v^{(j)}(\lambda)$.

$\left(S_{2}\right)$ :

$$
\left|g\left(s, u^{(0)}, \ldots, u^{(n-1)}\right)-g\left(s, v^{(0)}, \ldots, v^{(n-1)}\right)\right| \leq N \sum_{j=0}^{n-1}\left|u^{(j)}-v^{(j)}\right|,
$$

where $N>0$.

$\left(S_{3}\right)$ :

$$
\begin{aligned}
& \left|z\left(s, u^{(0)}, \ldots, u^{(n-1)}, \bar{u}^{(0)}, \ldots, \bar{u}^{(n-1)}, \tilde{u}\right)-z\left(s, v^{(0)}, \ldots, v^{(n-1)}, \bar{v}^{(0)}, \ldots, \bar{v}^{(n-1)}, \tilde{v}\right)\right| \\
& \leq e(s)\left|u^{(0)}-v^{(0)}\right|^{l}+k(s)\left|\bar{u}^{(0)}-\bar{v}^{(0)}\right|^{m}+M(s)|\tilde{u}-\tilde{v}|,
\end{aligned}
$$


where $e(s), k(s), M(s)>0, s \in J_{1} ; l, m \in(0,1] ; \bar{u}^{(0)}=u^{(0)}(\lambda), \bar{v}^{(0)}=v^{(0)}(\lambda)$.

$\left(S_{4}\right)$ :

$$
\left|g\left(s, u^{(0)}, \ldots, u^{(n-1)}\right)-g\left(s, v^{(0)}, \ldots, v^{(n-1)}\right)\right| \leq N(s)\left|u^{(0)}-v^{(0)}\right|^{n},
$$

where $N(s)>0, s \in J_{1} ; n \in(0,1]$.

$\left(S_{5}\right)$ :

Assume $\sigma(s)$ is a function from $J_{2}$ to $\mathbb{R}^{+}$and there exists $L_{\sigma}>0$ such that

$$
\int_{\tau}^{s} \sigma(r) d r \leq L_{\sigma} \cdot \sigma(s), s \in J_{2} .
$$

Firstly, we give the existence and uniqueness of a solution for (3).

Theorem 2. Assume that $\left(S_{1}\right)$ and $\left(S_{2}\right)$ hold. If $\frac{\eta^{n}}{(n-1) !}\left(2 K_{z}+M N \eta\right)<1$, then Equation (3) has a unique solution.

Proof. (i) We define the operator $\gamma$ as follows:

$$
(\gamma u)(s)=\left\{\begin{array}{l}
\sum_{j=0}^{n-1} \frac{\left(s-s_{0}\right)^{j} \chi^{(j)}\left(s_{0}\right)}{j !}+\frac{1}{(n-1) !} \int_{s_{0}}^{s}(s-\tau)^{n-1} \\
\cdot z\left(\tau, u^{(0)}, \ldots, u^{(n-1)}(\lambda), \int_{s_{0}}^{\tau} g\left(r, u^{(0)}, \ldots, u^{(n-1)}\right) d r\right) d \tau, s \in J_{2}, \\
\chi(s), s \in J_{3} .
\end{array}\right.
$$

Since $z \in C(B, \mathbb{R}), \gamma$ is well defined. Let $u_{1}(s), u_{2}(s) \in C\left(J_{1}, \mathbb{R}\right) \cap C^{n}\left(J_{2}, \mathbb{R}\right)$, for any $s \in J_{3}$. Then we have

$$
\left|\left(\gamma u_{1}\right)(s)-\left(\gamma u_{2}\right)(s)\right|=0 .
$$

For all $s \in J_{2}$, by condition $\left(S_{1}\right)$ and $\left(S_{2}\right)$, we have

$$
\begin{aligned}
& \left|\left(\gamma u_{1}\right)(s)-\left(\gamma u_{2}\right)(s)\right| \\
= & \mid \frac{1}{(n-1) !} \int_{s_{0}}^{s}(s-\tau)^{n-1}\left[z\left(\tau, u_{1}^{(0)}, \ldots, u_{1}^{(n-1)}(\lambda), \int_{s_{0}}^{\tau} g\left(r, u_{1}^{(0)}, \ldots, u_{1}^{(n-1)}\right) d r\right)\right. \\
& \left.-z\left(\tau, u_{2}^{(0)}, \ldots, u_{2}^{(n-1)}, u_{2}^{(0)}(\lambda), \ldots, u_{2}^{(n-1)}(\lambda), \int_{s_{0}}^{\tau} g\left(r, u_{2}^{(0)}, \ldots, u_{2}^{(n-1)}\right) d r\right)\right] d \tau \mid \\
\leq & \frac{1}{(n-1) !} \mid \int_{s_{0}}^{s}(s-\tau)^{n-1}\left[K_{z} \sum_{j=0}^{n-1}\left(\left|u_{1}^{(j)}-u_{2}^{(j)}\right|+\left|\bar{u}_{1}^{(j)}-\bar{u}_{2}^{(j)}\right|\right)\right. \\
& \left.+M N \int_{s_{0}}^{\tau} \sum_{j=0}^{n-1}\left|u_{1}^{(j)}-u_{2}^{(j)}\right| d r\right] d \tau \mid \\
\leq & \mid \int_{s_{0}}^{s} \frac{(s-\tau)^{n-1}}{(n-1) !}\left[K_{z} \sum_{j=0}^{n-1}\left(\sup \left|u_{1}^{(j)}-u_{2}^{(j)}\right|+\sup \left|\bar{u}_{1}^{(j)}-\bar{u}_{2}^{(j)}\right|\right)\right. \\
& \left.+M N \int_{s_{0}}^{\tau} \sum_{j=0}^{n-1} \sup \left|u_{1}^{(j)}-u_{2}^{(j)}\right| d r\right] d \tau \mid \\
\leq & \frac{1}{(n-1) !}\left|\int_{s_{0}}^{s}(s-\tau)^{n-1}\left(2 K_{z} n\left\|u_{1}-u_{2}\right\|+M N \eta n\left\|u_{1}-u_{2}\right\|\right) d \tau\right| \\
\leq & \frac{\eta^{n}}{(n-1) !}\left(2 K_{z}+M N \eta\right)\left\|u_{1}-u_{2}\right\|,
\end{aligned}
$$

where $\bar{u}_{i}^{(j)}=u_{i}^{(j)}(\lambda), i=1,2, j=0,1, \ldots, n$. 
Since $\frac{\eta^{n}}{(n-1) !}\left(2 K_{z}+M N \eta\right)<1$, for $u_{1}(s), u_{2}(s) \in C\left(J_{1}, \mathbb{R}\right) \cap C^{n}\left(J_{2}, \mathbb{R}\right)$, the operator $\gamma$ is a Banach contraction. By Banach contraction principle, the operator $\gamma$ has a unique fixed point $u^{*} \in C\left(J_{1}, \mathbb{R}\right) \cap C^{n}\left(J_{2}, \mathbb{R}\right)$; thus, Equation (3) has a unique solution.

Next, we obtain the following Ulam stability results.

Theorem 3. If the assumptions of the Theorem 2 are satisfied, Equation (3) is Hyers-Ulam stable on $J_{1}$.

Proof. Let $u(s) \in C\left(J_{1}, \mathbb{R}\right) \cap C^{n}\left(J_{2}, \mathbb{R}\right)$ be a unique solution of delay integro-differential equation

$$
\left\{\begin{array}{l}
u^{(n)}(s)=z\left(s, u^{(0)}, \ldots, u^{(0)}(\lambda), \ldots, u^{(n-1)}(\lambda), \int_{s_{0}}^{s} g\left(\tau, u^{(0)}, \ldots, u^{(n-1)}\right) d \tau\right), s \in J_{2} \\
u(s)=\chi(s), s \in J_{3} \\
u^{(j)}\left(s_{0}\right)=\chi^{(j)}\left(s_{0}\right), j=1, \ldots, n-1 .
\end{array}\right.
$$

Since $z \in C\left(J_{2} \times \mathbb{R}^{2 n+1}, \mathbb{R}\right), \lambda \in C\left(J_{2}, J_{1}\right)$, from Theorem 1 , we have

$$
u(s)=\left\{\begin{array}{l}
\sum_{j=0}^{n-1} \frac{\left(s-s_{0}\right)^{j} \chi^{(j)}\left(s_{0}\right)}{j !}+\frac{1}{(n-1) !} \int_{s_{0}}^{s}(s-\tau)^{n-1} \\
\cdot z\left(\tau, u^{(0)}, \ldots, u^{(0)}(\lambda), \ldots, u^{(n-1)}(\lambda), \int_{s_{0}}^{\tau} g\left(r, u^{(0)}, \ldots, u^{(n-1)}\right) d r\right) d \tau, s \in J_{2} \\
\chi(s), s \in J_{3} .
\end{array}\right.
$$

Let $v(s) \in C\left(J_{1}, \mathbb{R}\right) \cap C^{n}\left(J_{2}, \mathbb{R}\right)$ satisfy the following inequality:

$$
\left\{\begin{array}{l}
\left|v^{(n)}(s)-z\left(s, v^{(0)}, \ldots, v^{(n-1)}(\lambda), \int_{s_{0}}^{s} g\left(\tau, v^{(0)}, \ldots, v^{(n-1)}\right) d \tau\right)\right| \leq \theta, s \in J_{2}, \\
|v(s)-\chi(s)| \leq \theta, s \in J_{3} .
\end{array}\right.
$$

Let

$$
v^{(n)}(s)=z\left(s, v^{(0)}, \ldots, v^{(n-1)}(\lambda), \int_{s_{0}}^{s} g\left(\tau, v^{(0)}, \ldots, v^{(n-1)}\right) d \tau\right)+F(s),
$$

from (11), this implies that

$$
|F(s)| \leq \theta, s \in J_{2} \text {. }
$$

By Theorem 1, we have

$$
\begin{aligned}
v(s)= & \sum_{j=0}^{n-1} \frac{\left(s-s_{0}\right)^{j} \chi^{(j)}\left(s_{0}\right)}{j !}+\frac{1}{(n-1) !} \int_{s_{0}}^{s}(s-\tau)^{n-1} z\left(\tau, v^{(0)}, \ldots, v^{(n-1)}(\lambda),\right. \\
& \left.\int_{s_{0}}^{\tau} g\left(r, v^{(0)}, \ldots, v^{(n-1)}\right) d r\right) d \tau+\frac{1}{(n-1) !} \int_{s_{0}}^{s}(s-\tau)^{n-1} F(\tau) d \tau,
\end{aligned}
$$

then

$$
\begin{aligned}
& \mid v(s)-\sum_{j=0}^{n-1} \frac{\left(s-s_{0}\right)^{j} \chi^{(j)}\left(s_{0}\right)}{j !}-\frac{1}{(n-1) !} \int_{s_{0}}^{s}(s-\tau)^{n-1} z\left(\tau, v^{(0)}, \ldots, v^{(n-1)}(\lambda),\right. \\
& \left.\int_{s_{0}}^{\tau} g\left(r, v^{(0)}, \ldots, v^{(n-1)}\right) d r\right) d \tau \mid \\
= & \left|\frac{1}{(n-1) !} \int_{s_{0}}^{s}(s-\tau)^{n-1} F(\tau) d \tau\right|
\end{aligned}
$$




$$
\begin{aligned}
& \leq \frac{1}{(n-1) !} \int_{s_{0}}^{s}(s-\tau)^{n-1}|F(\tau)| d \tau \\
& \leq \frac{1}{(n-1) !} \int_{s_{0}}^{s}(s-\tau)^{n-1} d \tau \cdot \theta \\
& \leq \eta^{n} \theta .
\end{aligned}
$$

For all $s \in J_{3}$,

$$
|v(s)-u(s)|=0 .
$$

For any $s \in J_{2}$,

$$
\begin{aligned}
& |v(s)-u(s)| \\
& \leq \mid v(s)-\sum_{j=0}^{n-1} \frac{\left(s-s_{0}\right)^{j} \chi^{(j)}\left(s_{0}\right)}{j !}-\frac{1}{(n-1) !} \int_{s_{0}}^{s}(s-\tau)^{n-1} z\left(\tau, v^{(0)}, \ldots, v^{(n-1)}, v^{(0)}(\lambda),\right. \\
& \left.\ldots, v^{(n-1)}(\lambda), \int_{s_{0}}^{\tau} g\left(r, v^{(0)}, \ldots, v^{(n-1)}\right) d r\right) d \tau\left|+\frac{1}{(n-1) !} \int_{s_{0}}^{s}(s-\tau)^{n-1} \cdot\right| z\left(\tau, v^{(0)},\right. \\
& \left.\ldots, v^{(n-1)}, v^{(0)}(\lambda), \ldots, v^{(n-1)}(\lambda), \int_{s_{0}}^{\tau} g\left(r, v^{(0)}, \ldots, v^{(n-1)}\right) d r\right)-z\left(\tau, u^{(0)},\right. \\
& \left.\ldots, u^{(n-1)}, u^{(0)}(\lambda), \ldots, u^{(n-1)}(\lambda), \int_{s_{0}}^{\tau} g\left(r, u^{(0)}, \ldots, u^{(n-1)}\right) d r\right) \mid d \tau \\
& \leq \eta^{n} \theta+\frac{1}{(n-1) !} \int_{s_{0}}^{s}(s-\tau)^{n-1}\left[K_{z} \sum_{j=0}^{n-1}\left(\left|v^{(j)}-u^{(j)}\right|+\left|\bar{v}^{(j)}-\bar{u}^{(j)}\right|\right)\right. \\
& \left.\quad+M N \int_{s_{0}}^{\tau} \sum_{j=0}^{n-1}\left|v^{(j)}-u^{(j)}\right| d r\right] d \tau,
\end{aligned}
$$

where $\bar{u}^{(j)}=u^{(j)}(\lambda), \bar{v}^{(j)}=v^{(j)}(\lambda)$.

From the above inequality, we define the operator $A$ as follows: for all $s \in J_{2}$,

$$
\left(A y^{(j)}\right)(s)=\eta^{n} \theta+\frac{1}{(n-1) !} \int_{s_{0}}^{s}(s-\tau)^{n-1}\left[K_{z} \sum_{j=0}^{n-1}\left(y^{(j)}+\bar{y}^{(j)}\right)+M N \int_{s_{0}}^{\tau} \sum_{j=0}^{n-1} y^{(j)} d r\right] d \tau,
$$

for all $s \in J_{3}$,

$$
\left(A y^{(j)}\right)(s)=0
$$

where $\bar{y}^{(j)}=y^{(j)}(\lambda), j=0,1, \ldots, n$.

For all $s \in J_{2}$,

$$
\begin{aligned}
& \left|\left(A y_{1}{ }^{(j)}\right)(s)-\left(A y_{2}{ }^{(j)}\right)(s)\right| \\
\leq & \frac{1}{(n-1) !} \int_{s_{0}}^{s}(s-\tau)^{n-1}\left[K_{z} \sum_{j=0}^{n-1}\left(\left|y_{1}^{(j)}-y_{2}{ }^{(j)}\right|+\left|\bar{y}_{1}^{(j)}-\bar{y}_{2}{ }^{(j)}\right|\right)\right. \\
& \left.+M N \int_{s_{0}}^{\tau} \sum_{j=0}^{n-1}\left|y_{1}^{(j)}-y_{2}^{(j)}\right| d r\right] d \tau \\
\leq & \frac{1}{(n-1) !} \int_{s_{0}}^{s}(s-\tau)^{n-1} d s\left(2 K_{z} n+M N \eta n\right)\left\|y_{1}{ }^{(j)}-y_{2}{ }^{(j)}\right\| \\
\leq & \frac{\eta^{n}}{(n-1) !}\left(2 K_{z}+M N \eta\right)\left\|y_{1}{ }^{(j)}-y_{2}{ }^{(j)}\right\|,
\end{aligned}
$$


where $\bar{y}_{i}^{(j)}=y_{i}^{(j)}(\lambda), i=1,2, j=0,1, \ldots, n$.

Since

$$
\frac{\eta^{n}}{(n-1) !}\left(2 K_{z}+M N \eta\right)<1
$$

$A$ is a strict contraction operator.

By the contraction mapping theorem, $A$ has a unique fixed point $\left\{\omega^{(j)}\right\}$, so

$$
\omega^{(j)}(s)=\eta^{n} \theta+\frac{1}{(n-1) !} \int_{s_{0}}^{s}(s-\tau)^{n-1}\left[K_{z} \sum_{j=0}^{n-1}\left(\omega^{(j)}+\bar{\omega}^{(j)}\right)+M N \int_{s_{0}}^{\tau} \sum_{j=0}^{n-1} \omega^{(j)} d r\right] d \tau, s \in J_{2} .
$$

Since $\left(\omega^{(j)}(s)\right)^{\prime} \geq 0, \omega^{(j)}(s)$ is a nondecreasing function, we have

$$
\begin{gathered}
\omega^{(j)}(\lambda(s))=\bar{\omega}^{(j)}(s) \leq \omega^{(j)}(s), \\
\omega^{(j)}(s) \leq \eta^{n} \theta+\frac{1}{(n-1) !} \int_{s_{0}}^{s}(s-\tau)^{n-1}\left(2 K_{z} \sum_{j=0}^{n-1} \omega^{(j)}+M N \int_{s_{0}}^{\tau} \sum_{j=0}^{n-1} \omega^{(j)} d r\right) d \tau \\
\leq \eta^{n} \theta+\frac{1}{(n-1) !} \sum_{j=0}^{n-1}\left(2 K_{z}+M N \eta\right) \int_{s_{0}}^{s}(s-\tau)^{n-1} \omega^{(j)} d \tau \\
\leq \eta^{n} \theta+\frac{n}{(n-1) !}\left(2 K_{z}+M N \eta\right) \int_{s_{0}}^{s}(s-\tau)^{n-1} \omega^{(j)} d \tau .
\end{gathered}
$$

From Lemma 3, we obtain

$$
\omega^{(j)}(s) \leq C \cdot \theta, C=\eta^{n} \exp \left(\frac{\left(2 K_{z}+M N \eta\right) \eta^{n}}{(n-1) !}\right) .
$$

Since

$$
|v(s)-u(s)| \leq\left(A y^{(j)}\right)(s)
$$

then

$$
|v(s)-u(s)| \leq\left(A w^{(j)}\right)(s)=w^{(j)}(s) \leq C \cdot \theta .
$$

From Definition 1, Equation (3) is Hyers-Ulam stable.

Theorem 4. Assume that $\left(S_{3}\right)$ and $\left(S_{4}\right)$ hold; then Equation (3) is Hyers-Ulam stable on $J_{1}$.

Proof. Let $u(s) \in C\left(J_{1}, \mathbb{R}\right) \cap C^{n}\left(J_{2}, \mathbb{R}\right)$ be a solution of delay integro-differential equation

$$
\left\{\begin{array}{l}
u^{(n)}(s)=z\left(s, u^{(0)}, \ldots, u^{(0)}(\lambda), \ldots, u^{(n-1)}(\lambda), \int_{s_{0}}^{s} g\left(\tau, u^{(0)}, \ldots, u^{(n-1)}\right) d \tau\right), s \in J_{2}, \\
u(s)=\chi(s), s \in J_{3}, \\
u^{(j)}\left(s_{0}\right)=\chi^{(j)}\left(s_{0}\right), j=1, \ldots, n-1 .
\end{array}\right.
$$

Since $z \in C\left(J_{2} \times \mathbb{R}^{2 n+1}, \mathbb{R}\right), \lambda \in C\left(J_{2}, J_{1}\right)$, from Theorem 1 , we have

$$
u(s)=\left\{\begin{array}{l}
\sum_{j=0}^{n-1} \frac{\left(s-s_{0}\right)^{j} \chi^{(j)}\left(s_{0}\right)}{j !}+\frac{1}{(n-1) !} \int_{s_{0}}^{s}(s-\tau)^{n-1} \\
\cdot z\left(\tau, u^{(0)}, \ldots, u^{(0)}(\lambda), \ldots, u^{(n-1)}(\lambda), \int_{s_{0}}^{\tau} g\left(r, u^{(0)}, \ldots, u^{(n-1)}\right) d r\right) d \tau, s \in J_{2}, \\
\chi(s), s \in J_{3} .
\end{array}\right.
$$


Let $v(s) \in C\left(J_{1}, \mathbb{R}\right) \cap C^{n}\left(J_{2}, \mathbb{R}\right)$ satisfying inequality

$\left\{\begin{array}{l}\left|v^{(n)}(s)-z\left(s, v^{(0)}, \ldots, v^{(n-1)}(\lambda), \int_{s_{0}}^{s} g\left(\tau, v^{(0)}, \ldots, v^{(n-1)}\right) d \tau\right)\right| \leq \theta, s \in J_{2}, \\ |v(s)-\chi(s)| \leq \theta, s \in J_{3} .\end{array}\right.$

Let

$$
v^{(n)}(s)=z\left(s, v^{(0)}, \ldots, v^{(n-1)}(\lambda), \int_{s_{0}}^{s} g\left(\tau, v^{(0)}, \ldots, v^{(n-1)}\right) d \tau\right)+F(s),
$$

from (14), this implies that

$$
|F(s)| \leq \theta, s \in J_{2} .
$$

By Theorem 1, we have

$$
\begin{aligned}
v(s)= & \sum_{j=0}^{n-1} \frac{\left(s-s_{0}\right)^{j} \chi^{(j)}\left(s_{0}\right)}{j !}+\frac{1}{(n-1) !} \int_{s_{0}}^{s}(s-\tau)^{n-1} z\left(\tau, v^{(0)}, \ldots, v^{(n-1)}(\lambda),\right. \\
& \left.\int_{s_{0}}^{\tau} g\left(r, v^{(0)}, \ldots, v^{(n-1)}\right) d r\right) d \tau+\frac{1}{(n-1) !} \int_{s_{0}}^{s}(s-\tau)^{n-1} F(\tau) d \tau,
\end{aligned}
$$

then

$$
\begin{aligned}
& \mid v(s)-\sum_{j=0}^{n-1} \frac{\left(s-s_{0}\right)^{j} \chi^{(j)}\left(s_{0}\right)}{j !}-\frac{1}{(n-1) !} \int_{s_{0}}^{s}(s-\tau)^{n-1} z\left(\tau, v^{(0)}, \ldots, v^{(n-1)}(\lambda),\right. \\
& \left.\int_{s_{0}}^{\tau} g\left(r, v^{(0)}, \ldots, v^{(n-1)}\right) d r\right) d \tau \mid \\
= & \left|\frac{1}{(n-1) !} \int_{s_{0}}^{s}(s-\tau)^{n-1} F(\tau) d \tau\right| \\
\leq & \frac{1}{(n-1) !} \int_{s_{0}}^{s}(s-\tau)^{n-1}|F(\tau)| d \tau \\
\leq & \frac{1}{(n-1) !} \int_{s_{0}}^{s}(s-\tau)^{n-1} d \tau \cdot \theta \\
\leq & \eta^{n} \theta .
\end{aligned}
$$

For all $s \in J_{3}$,

$$
|v(s)-u(s)|=0 .
$$

For any $s \in J_{2}$,

$$
\begin{aligned}
& |v(s)-u(s)| \\
= & \mid v(s)-\sum_{j=0}^{n-1} \frac{\left(s-s_{0}\right)^{j} \chi^{(j)}\left(s_{0}\right)}{j !}-\frac{1}{(n-1) !} \int_{s_{0}}^{s}(s-\tau)^{n-1} z\left(\tau, u^{(0)}, \ldots, u^{(n-1)}, u^{(0)}(\lambda),\right. \\
& \left.\ldots, u^{(n-1)}(\lambda), \int_{s_{0}}^{\tau} g\left(r, u^{(0)}, \ldots, u^{(n-1)}\right) d r\right) d \tau \mid \\
= & \mid v(s)-\sum_{j=0}^{n-1} \frac{\left(s-s_{0}\right)^{j} \chi^{(j)}\left(s_{0}\right)}{j !}-\frac{1}{(n-1) !} \int_{s_{0}}^{s}(s-\tau)^{n-1} z\left(\tau, v^{(0)}, \ldots, v^{(n-1)}, v^{(0)}(\lambda),\right. \\
& \left.\ldots, v^{(n-1)}(\lambda), \int_{s_{0}}^{\tau} g\left(r, v^{(0)}, \ldots, v^{(n-1)}\right) d r\right) d \tau|+| \frac{1}{(n-1) !} \int_{s_{0}}^{s}(s-\tau)^{n-1} z\left(\tau, v^{(0)},\right.
\end{aligned}
$$




$$
\begin{aligned}
& \left.\ldots, v^{(n-1)}, v^{(0)}(\lambda), \ldots, v^{(n-1)}(\lambda), \int_{s_{0}}^{\tau} g\left(r, v^{(0)}, \ldots, v^{(n-1)}\right) d r\right) d \tau \\
& -\frac{1}{(n-1) !} \int_{s_{0}}^{s}(s-\tau)^{n-1} z\left(\tau, u^{(0)}, \ldots, u^{(n-1)}, u^{(0)}(\lambda)\right. \\
& \left.\ldots, u^{(n-1)}(\lambda), \int_{s_{0}}^{\tau} g\left(r, u^{(0)}, \ldots, u^{(n-1)}\right) d r\right) d \tau \mid \\
& \leq \mid v(s)-\sum_{j=0}^{n-1} \frac{\left(s-s_{0}\right)^{j} \chi^{(j)}\left(s_{0}\right)}{j !}-\frac{1}{(n-1) !} \int_{s_{0}}^{s}(s-\tau)^{n-1} z\left(\tau, v^{(0)}, \ldots, v^{(n-1)},\right. \\
& \left.\quad v^{(0)}(\lambda), \ldots, v^{(n-1)}(\lambda), \int_{s_{0}}^{\tau} g\left(r, v^{(0)}, \ldots, v^{(n-1)}\right) d r\right) d \tau \mid \\
& +\mid \frac{1}{(n-1) !} \int_{s_{0}}^{s}(s-\tau)^{n-1}\left[e(\tau)\left|v^{(0)}-u^{(0)}\right|^{l}+k(\tau)\left|v^{(0)}(\lambda)-u^{(0)}(\lambda)\right|^{m}\right. \\
& \left.+\int_{s_{0}}^{\tau} M(\tau) N(r)\left|v^{(0)}-u^{(0)}\right|^{n} d r\right] d \tau \mid,
\end{aligned}
$$

then

$$
\begin{aligned}
|v(s)-u(s)| \leq & \eta^{n} \theta+\frac{1}{(n-1) !} \int_{s_{0}}^{s}(s-\tau)^{n-1}\left[e(\tau)|v-u|^{l}+k(\tau)|v(\lambda)-u(\lambda)|^{m}\right. \\
& \left.+\int_{s_{0}}^{\tau} M(\tau) N(r)|v-u|^{n} d r\right] d \tau .
\end{aligned}
$$

From Lemma 4 , set $h=1, a=m, b=l, c=n, w(s)=|v(s)-u(s)|, d(s)=\eta^{n} \theta$, $l(s)=\frac{1}{(n-1) !}, m(\tau)=(s-\tau)^{n-1} k(\tau), n(\tau)=(s-\tau)^{n-1} e(\tau), z(r)=(s-\tau)^{n-1} M(\tau) N(r)$; we have

$$
\begin{gathered}
|v(s)-u(s)| \leq \eta^{n} \theta+\frac{1}{(n-1) !} U(s) \exp \left(\int_{s_{0}}^{s} V(\tau) d \tau\right), \\
|v(s)-u(s)| \leq\left[\eta^{n}+\frac{1}{(n-1) !} \frac{U(s)}{\theta} \exp \left(\int_{s_{0}}^{s} V(\tau) d \tau\right)\right] \cdot \theta \leq K_{z, \theta} \cdot \theta,
\end{gathered}
$$

where for any $H>0$,

$$
\begin{aligned}
& K_{z, \theta}=\max _{s \in J_{1}}\left\{\eta^{n}+\frac{1}{(n-1) !} \frac{U(s)}{\theta} \exp \left(\int_{s_{0}}^{s} V(\tau) d \tau\right)\right\} \\
& U(s)=\int_{s_{0}}^{s}\left[(s-\tau)^{n-1} k(\tau)\left(m H^{m-1} \eta^{n} \theta+(1-m) H^{m}\right)+g(\tau, s)\left(l H^{l-1} \eta^{n} \theta+(1-l) H^{l}\right)\right. \\
& \left.+\int_{s_{0}}^{\tau}(s-\tau)^{n-1} M(\tau) N(r)\left(n H^{n-1} \eta^{n} \theta+(1-n) H^{n}\right) d r\right] d \tau, \\
& V(s)=\frac{1}{(n-1) !} \int_{s_{0}}^{s}(s-\tau)^{n-1} M(\tau) N(\tau) n H^{n-1} d \tau \text {. }
\end{aligned}
$$

From Definition 1, Equation (3) is Hyers-Ulam stable.

Theorem 5. Assume that $\left(S_{3}\right),\left(S_{4}\right)$ and $\left(S_{5}\right)$ hold; then Equation (3) is Hyers-Ulam-Rassias stable with respect to $\sigma(s)$ on $J_{1}$.

Proof. Let $u(s) \in C\left(J_{1}, \mathbb{R}\right) \cap C^{n}\left(J_{2}, \mathbb{R}\right)$ be a unique solution of delay integro-differential equation 


$$
\left\{\begin{array}{l}
u^{(n)}(s)=z\left(s, u^{(0)}, \ldots, u^{(0)}(\lambda), \ldots, u^{(n-1)}(\lambda), \int_{s_{0}}^{s} g\left(\tau, u^{(0)}, \ldots, u^{(n-1)}\right) d \tau\right), s \in J_{2}, \\
u(s)=\chi(s), s \in J_{3} \\
u^{(j)}\left(s_{0}\right)=\chi^{(j)}\left(s_{0}\right), j=1, \ldots, n-1 .
\end{array}\right.
$$

Since $z \in C\left(J_{2} \times \mathbb{R}^{2 n+1}, \mathbb{R}\right), \lambda \in C\left(J_{2}, J_{1}\right)$, from Theorem 1 , we have

$$
u(s)=\left\{\begin{array}{l}
\sum_{j=0}^{n-1} \frac{\left(s-s_{0}\right)^{j} \chi^{(j)}\left(s_{0}\right)}{j !}+\frac{1}{(n-1) !} \int_{s_{0}}^{s}(s-\tau)^{n-1} \\
z\left(\tau, u^{(0)}, \ldots, u^{(0)}(\lambda), \ldots, u^{(n-1)}(\lambda), \int_{s_{0}}^{\tau} g\left(r, u^{(0)}, \ldots, u^{(n-1)}\right) d r\right) d \tau, s \in J_{2}, \\
\chi(s), s \in J_{3} .
\end{array}\right.
$$

Let $v(s) \in C\left(J_{1}, \mathbb{R}\right) \cap C^{n}\left(J_{2}, \mathbb{R}\right)$ satisfy the following inequality

$$
\left\{\begin{array}{l}
\left|v^{(n)}(s)-z\left(s, v^{(0)}, \ldots, v^{(n-1)}(\lambda), \int_{s_{0}}^{s} g\left(\tau, v^{(0)}, \ldots, v^{(n-1)}\right) d \tau\right)\right| \leq \sigma(s), s \in J_{2}, \\
|v(s)-\chi(s)| \leq \sigma(s), s \in J_{3} .
\end{array}\right.
$$

Let

$$
v^{(n)}(s)=z\left(s, v^{(0)}, \ldots, v^{(n-1)}(\lambda), \int_{s_{0}}^{s} g\left(\tau, v^{(0)}, \ldots, v^{(n-1)}\right) d \tau\right)+F(s),
$$

from (17), this implies that

$$
|F(s)| \leq \sigma(s), s \in J_{2} .
$$

By Theorem 1, we have

$$
\begin{aligned}
v(s)= & \sum_{j=0}^{n-1} \frac{\left(s-s_{0}\right)^{j} \chi^{(j)}\left(s_{0}\right)}{j !}+\frac{1}{(n-1) !} \int_{s_{0}}^{s}(s-\tau)^{n-1} z\left(\tau, v^{(0)}, \ldots, v^{(n-1)}(\lambda),\right. \\
& \left.\int_{s_{0}}^{\tau} g\left(r, v^{(0)}, \ldots, v^{(n-1)}\right) d r\right) d \tau+\frac{1}{(n-1) !} \int_{s_{0}}^{s}(s-\tau)^{n-1} F(\tau) d \tau ;
\end{aligned}
$$

then

$$
\begin{aligned}
& \mid v(s)-\sum_{j=0}^{n-1} \frac{\left(s-s_{0}\right)^{j} \chi^{(j)}\left(s_{0}\right)}{j !}-\frac{1}{(n-1) !} \int_{s_{0}}^{s}(s-\tau)^{n-1} z\left(\tau, v^{(0)}, \ldots, v^{(n-1)}(\lambda),\right. \\
& \left.\int_{s_{0}}^{\tau} g\left(r, v^{(0)}, \ldots, v^{(n-1)}\right) d r\right) d \tau \mid \\
= & \left|\frac{1}{(n-1) !} \int_{s_{0}}^{s}(s-\tau)^{n-1} F(\tau) d \tau\right| \\
\leq & \frac{1}{(n-1) !} \int_{s_{0}}^{s}(s-\tau)^{n-1}|F(\tau)| d \tau \\
\leq & \frac{1}{(n-1) !} \int_{s_{0}}^{s}(s-\tau)^{n-1} \sigma(\tau) d \tau .
\end{aligned}
$$

By Lemma 1 and condition $\left(S_{5}\right)$, we obtain

$$
\begin{aligned}
& \mid v(s)-\sum_{j=0}^{n-1} \frac{\left(s-s_{0}\right)^{j} \chi^{(j)}\left(s_{0}\right)}{j !}-\frac{1}{(n-1) !} \int_{s_{0}}^{s}(s-\tau)^{n-1} z\left(\tau, v^{(0)}, \ldots, v^{(n-1)}(\lambda),\right. \\
& \left.\int_{s_{0}}^{\tau} g\left(r, v^{(0)}, \ldots, v^{(n-1)}\right) d r\right) d \tau \mid
\end{aligned}
$$




$$
\begin{aligned}
& \leq \int_{s_{0}}^{s} \int_{s_{0}}^{s_{1}} \int_{s_{0}}^{s_{2}} \int_{s_{0}}^{s_{3}} \ldots \int_{s_{0}}^{s_{n-1}} \sigma\left(s_{n}\right) d s_{n} d s_{n-1} \ldots d s_{2} d s_{1} \\
& \leq L_{\sigma}{ }^{n} \cdot \sigma(s) .
\end{aligned}
$$

For all $s \in J_{3}$,

$$
|v(s)-u(s)|=0
$$

For any $s \in J_{2}$,

$$
\begin{aligned}
& |v(s)-u(s)| \\
& =\mid v(s)-\sum_{j=0}^{n-1} \frac{\left(s-s_{0}\right)^{j} \chi^{(j)}\left(s_{0}\right)}{j !}-\frac{1}{(n-1) !} \int_{s_{0}}^{s}(s-\tau)^{n-1} z\left(\tau, u^{(0)}, \ldots, u^{(n-1)}, u^{(0)}(\lambda),\right. \\
& \left.\ldots, u^{(n-1)}(\lambda), \int_{s_{0}}^{\tau} g\left(r, u^{(0)}, \ldots, u^{(n-1)}\right) d r\right) d \tau \\
& =\mid v(s)-\sum_{j=0}^{n-1} \frac{\left(s-s_{0}\right)^{j} \chi^{(j)}\left(s_{0}\right)}{j !}-\frac{1}{(n-1) !} \int_{s_{0}}^{s}(s-\tau)^{n-1} z\left(\tau, v^{(0)}, \ldots, v^{(n-1)}, v^{(0)}(\lambda),\right. \\
& \left.\ldots, v^{(n-1)}(\lambda), \int_{s_{0}}^{\tau} g\left(r, v^{(0)}, \ldots, v^{(n-1)}\right) d r\right) d \tau|+| \frac{1}{(n-1) !} \int_{s_{0}}^{s}(s-\tau)^{n-1} z\left(\tau, v^{(0)}\right. \text {, } \\
& \left.\ldots, v^{(n-1)}, v^{(0)}(\lambda), \ldots, v^{(n-1)}(\lambda), \int_{s_{0}}^{\tau} g\left(r, v^{(0)}, \ldots, v^{(n-1)}\right) d r\right) d \tau \\
& -\frac{1}{(n-1) !} \int_{s_{0}}^{s}(s-\tau)^{n-1} z\left(\tau, u^{(0)}, \ldots, u^{(n-1)}, u^{(0)}(\lambda),\right. \\
& \left.\ldots, u^{(n-1)}(\lambda), \int_{s_{0}}^{\tau} g\left(r, u^{(0)}, \ldots, u^{(n-1)}\right) d r\right) d \tau \\
& \leq \mid v(s)-\sum_{j=0}^{n-1} \frac{\left(s-s_{0}\right)^{j} \chi^{(j)}\left(s_{0}\right)}{j !}-\frac{1}{(n-1) !} \int_{s_{0}}^{s}(s-\tau)^{n-1} z\left(\tau, v^{(0)}, \ldots, v^{(n-1)}, v^{(0)}(\lambda),\right. \\
& \left.\ldots, v^{(n-1)}(\lambda), \int_{s_{0}}^{\tau} g\left(r, v^{(0)}, \ldots, v^{(n-1)}\right) d r\right) d \tau \mid \\
& +\mid \frac{1}{(n-1) !} \int_{s_{0}}^{s}(s-\tau)^{n-1}\left[e(\tau)\left|v^{(0)}-u^{(0)}\right|^{l}+k(\tau)\left|v^{(0)}(\lambda)-u^{(0)}(\lambda)\right|^{m}\right. \\
& \left.+\int_{s_{0}}^{\tau} M(\tau) N(r)\left|v^{(0)}-u^{(0)}\right|^{n} d r\right] d \tau \mid
\end{aligned}
$$

then

$$
\begin{aligned}
& |v(s)-u(s)| \\
\leq & L_{\sigma}^{n} \sigma(s)+\frac{1}{(n-1) !} \int_{s_{0}}^{s}(s-\tau)^{n-1}\left[e(\tau)|v-u|^{l}+k(\tau)|v(\lambda)-u(\lambda)|^{m}\right. \\
& \left.+\int_{s_{0}}^{\tau} M(\tau) N(r)|v-u|^{n} d r\right] d \tau .
\end{aligned}
$$

From Lemma 4, set $h=1, a=m, b=l, c=n, w(s)=|v(s)-u(s)|, d(s)=L_{\sigma}{ }^{n} \sigma(s)$, $l(s)=\frac{1}{(n-1) !}, m(\tau)=(s-\tau)^{n-1} k(\tau), n(\tau)=(s-\tau)^{n-1} e(\tau), z(r)=(s-\tau)^{n-1} M(\tau) N(r)$; thus, we have

$$
|v(s)-u(s)| \leq L_{\sigma}{ }^{n} \sigma(s)+\frac{1}{(n-1) !} U(s) \exp \left(\int_{s_{0}}^{s} V(\tau) d \tau\right)
$$




$$
|v(s)-u(s)| \leq\left[L_{\sigma}{ }^{n}+\frac{1}{(n-1) !} \frac{U(s)}{\sigma(s)} \exp \left(\int_{s_{0}}^{s} V(\tau) d \tau\right)\right] \cdot \sigma(s) \leq K_{\sigma} \cdot \sigma(s),
$$

where for any $H>0$,

$$
\begin{gathered}
K_{\sigma}=\max _{s \in J_{1}}\left\{L_{\sigma}{ }^{n}+\frac{1}{(n-1) !} \frac{U(s)}{\sigma(s)} \exp \left(\int_{s_{0}}^{s} V(\tau) d \tau\right)\right\}, \\
U(s)=\int_{s_{0}}^{s}\left[(s-\tau)^{n-1} k(\tau)\left(m H^{m-1} L_{\sigma}{ }^{n} \sigma(\tau)+(1-m) H^{m}\right)+g(\tau, s)\left(l H^{l-1} L_{\sigma}{ }^{n} \sigma(\tau)\right.\right. \\
\left.\left.+(1-l) H^{l}\right)+\int_{s_{0}}^{\tau}(s-\tau)^{n-1} M(\tau) N(r)\left(n H^{n-1} L_{\sigma}{ }^{n} \sigma(r)+(1-n) H^{n}\right) d r\right] d \tau, \\
V(s)=\frac{1}{(n-1) !} \int_{s_{0}}^{s}(s-\tau)^{n-1} M(\tau) N(\tau) n H^{n-1} d \tau .
\end{gathered}
$$

From Definition 2, Equation (3) is Hyers-Ulam-Rassias stable.

\section{Examples}

Example 1. We consider the delay integro-differential equation

$$
\frac{d u(s)}{d s}=e^{s}\left[\frac{1}{|u(s)|+8}+\frac{1}{|u(\lambda(s))|+8}+\frac{1}{64} \int_{0}^{s} e^{-\tau} \frac{1}{|u(\tau)|+8} d \tau\right]
$$

and the inequality

$$
\left|\frac{d u(s)}{d s}-e^{s}\left[\frac{1}{|u(s)|+8}+\frac{1}{|u(\lambda(s))|+8}+\frac{1}{64} \int_{0}^{s} e^{-\tau} \frac{1}{|u(\tau)|+8} d \tau\right]\right| \leq \sigma(s) .
$$

Set $z(s, u(s), u(\lambda(s)), v)=e^{s}\left[\frac{1}{|u(s)|+8}+\frac{1}{|u(\lambda(s))|+8}+\frac{1}{64} v\right], g(s, u(s))=e^{-s} \frac{1}{|u(s)|+8}$, $s \in[0,2]$.

For any $s \in[0,2]$, we obtain

$$
\begin{aligned}
& \left|z\left(s, u_{1}(s), u_{1}(\lambda(s)), v_{1}\right)-z\left(s, u_{2}(s), u_{2}(\lambda(s)), v_{2}\right)\right| \\
= & e^{s}\left|\frac{\left(\left|u_{2}(s)\right|-\left|u_{1}(s)\right|\right)}{\left(\left|u_{1}(s)\right|+8\right)\left(\left|u_{2}(s)\right|+8\right)}+\frac{\left(\left|u_{2}(\lambda(s))\right|-\left|u_{1}(\lambda(s))\right|\right)}{\left(\left|u_{1}(\lambda(s))\right|+8\right)\left(\left|u_{2}(\lambda(s))\right|+8\right)}+\frac{1}{64}\left(v_{1}-v_{2}\right)\right| \\
\leq & \frac{e^{s}}{64}\left|u_{1}(s)-u_{2}(s)\right|+\frac{e^{s}}{64}\left|u_{1}(\lambda(s))-u_{2}(\lambda(s))\right|+\frac{1}{64}\left|v_{1}(s)-v_{2}(s)\right| .
\end{aligned}
$$

Here, $e(s)=\frac{e^{s}}{64}, k(s)=\frac{e^{s}}{64}, M(s)=\frac{e^{s}}{64}, l=m=1$.

$$
\left|g\left(s, u_{1}(s)\right)-g\left(s, u_{2}(s)\right)\right|=e^{-s}\left|\frac{\left|u_{2}(s)\right|-\left|u_{1}(s)\right|}{\left(\left|u_{1}(s)\right|+8\right)\left(\left|u_{2}(s)\right|+8\right)}\right| \leq \frac{e^{-s}}{64}\left|u_{1}(s)-u_{2}(s)\right| .
$$

Here, $N(s)=\frac{e^{s}}{64}, n=1$.

Thus, $\left(S_{1}\right)$ and $\left(S_{2}\right)$ hold, $\frac{\eta^{n}}{(n-1) !}\left(2 K_{z}+M N \eta\right)=\frac{23}{50}<1$. From Theorem 2, Equation has a unique solution

$$
u(s)=\int_{0}^{s} e^{\tau}\left[\frac{1}{|u(\tau)|+8}+\frac{1}{|u(\lambda(\tau))|+8}+\frac{1}{64} \int_{0}^{\tau} e^{-t} \frac{1}{|u(t)|+8} d t\right] d \tau .
$$

Let $\sigma(s)=e^{s}, \int_{0}^{s} \sigma(\tau)=\int_{0}^{s} e^{\tau}=e^{s}-1 \leq e^{s}$, we have $L_{\sigma}=1>0$.

As v(s) satisfies the inequality

$$
\left|\frac{d v(s)}{d s}-e^{s}\left[\frac{1}{|v(s)|+8}+\frac{1}{|v(\lambda(s))|+8}+\frac{1}{64} \int_{0}^{s} e^{-\tau} \frac{1}{|v(\tau)|+8} d \tau\right]\right| \leq e^{s},
$$


we have

$$
\left|v(s)-\int_{0}^{s} e^{\tau}\left[\frac{1}{|v(\tau)|+8}+\frac{1}{|v(\lambda(\tau))|+8}+\frac{1}{64} \int_{0}^{\tau} e^{-t} \frac{1}{|v(t)|+8} d t\right] d \tau\right| \leq e^{s} .
$$

Since $\left(S_{3}\right),\left(S_{4}\right)$ and $\left(S_{5}\right)$ hold, from Theorem 5 , we have

$$
\begin{aligned}
& |v(s)-u(s)| \\
\leq & e^{s}+\int_{0}^{s}\left[\frac{e^{\tau}}{64}|v(\tau)-u(\tau)|+\frac{e^{\tau}}{64}|v(\lambda(\tau))-u(\lambda(\tau))|+\int_{0}^{\tau} \frac{e^{-r}}{64^{2}}|v(r)-u(r)| d r\right] d \tau \\
\leq & \frac{57}{500} e^{s} .
\end{aligned}
$$

Hence, the equation is Hyers-Ulam-Rassias stable.

Example 2. Consider the equation $[0,2]$.

$$
\frac{d u(s)}{d s}=-\frac{1}{8} \frac{1}{|u(s)|+1}-\frac{1}{8} \frac{1}{|u(\lambda(s))|+1}-\frac{1}{64} \int_{0}^{s} \frac{1}{|u(\tau)|+1} d \tau
$$

Set $z(s, u(s), u(\lambda(s)), v)=-\frac{1}{8} \frac{1}{|u(s)|+1}-\frac{1}{8} \frac{1}{|u(\lambda(s))|+1}-\frac{1}{64} v, g(s, u(s))=\frac{1}{|u(\tau)|+1}, s \in$

For any $s \in[0,2]$, we obtain

$$
\begin{aligned}
& \left|z\left(s, u_{1}(s), u_{1}(\lambda(s)), v_{1}\right)-z\left(s, u_{2}(s), u_{2}(\lambda(s)), v_{2}\right)\right| \\
= & \frac{1}{8}\left|\frac{\left(\left|u_{2}(s)\right|-\left|u_{1}(s)\right|\right)}{\left(\left|u_{1}(s)\right|+1\right)\left(\left|u_{2}(s)\right|+1\right)}+\frac{1}{8} \frac{\left(\left|u_{2}(\lambda(s))\right|-\left|u_{1}(\lambda(s))\right|\right)}{\left(\left|u_{1}(\lambda(s))\right|+1\right)\left(\left|u_{2}(\lambda(s))\right|+1\right)}+\frac{1}{64}\left(v_{2}-v_{1}\right)\right| \\
\leq & \frac{1}{8}\left|u_{1}(s)-u_{2}(s)\right|+\frac{1}{8}\left|u_{1}(\lambda(s))-u_{2}(\lambda(s))\right|+\frac{1}{64}\left|v_{1}(s)-v_{2}(s)\right|, \\
& \left|g\left(s, u_{1}(s)\right)-g\left(s, u_{2}(s)\right)\right|=\left|\frac{\left|u_{2}(s)\right|-\left|u_{1}(s)\right|}{\left(\left|u_{1}(s)\right|+1\right)\left(\left|u_{2}(s)\right|+1\right)}\right| \leq\left|u_{1}(s)-u_{2}(s)\right| .
\end{aligned}
$$

Thus, $\left(S_{1}\right)$ and $\left(S_{2}\right)$ hold, $\frac{\eta^{n}}{(n-1) !}\left(2 K_{z}+M N \eta\right)=\frac{9}{16}<1$. From Theorem 2 , the equation has a unique solution:

$$
u(s)=-\int_{0}^{s} \frac{1}{8}\left[\frac{1}{|u(\tau)|+1}+\frac{1}{8} \frac{1}{|u(\lambda(\tau))|+1}+\frac{1}{64} \int_{0}^{\tau} \frac{1}{|u(t)|+1} d t\right] d \tau
$$

Let $v(s)=e^{s}$ and choose $\theta=\frac{9}{32}$. We have

$$
\begin{aligned}
& \left|\frac{d v(s)}{d s}-\left[-\frac{1}{8} \frac{1}{|v(s)|+1}-\frac{1}{8} \frac{1}{|v(\lambda(s))|+1}-\frac{1}{64} \int_{0}^{s} \frac{1}{|v(\tau)|+1} d \tau\right]\right| \\
= & \left|\frac{1}{8} \frac{1}{|v(s)|+1}+\frac{1}{8} \frac{1}{|v(\lambda(s))|+1}+\frac{1}{64} \int_{0}^{s} \frac{1}{|v(\tau)|+1} d \tau-e^{-s}\right| \leq \frac{9}{32}=\theta .
\end{aligned}
$$

Since $\left(S_{3}\right)$ and $\left(S_{4}\right)$ hold, from Theorem 4 , we have

$$
\begin{aligned}
& |v(s)-u(s)| \\
\leq & 2 \theta+\int_{0}^{s}\left[\frac{1}{8}|v(\tau)-u(\tau)|+\frac{1}{8}|v(\lambda(\tau))-u(\lambda(\tau))|+\int_{0}^{\tau} \frac{1}{64}|v(r)-u(r)| d r\right] d \tau \\
\leq & \frac{16}{5} \theta .
\end{aligned}
$$

Hence, the equation is Hyers-Ulam stable. 


\section{Conclusions}

Based on Gronwall-Bellman inequality, we have proved the Ulam stability of nth order delay integro-differential equations. By applying Lipschitz conditions and the Banach contraction principle, the existence and uniqueness theorem of a solution was given. In addition, the expression of the solution played a great role in the proof of the main theorems. The Ulam stability of the $n$-th order delay integro-differential equation is related to many applications, such as the effect of tire dynamics on vehicle shimmy and optimal control of a size-structured population, and the research in this field is still open. In future work, we recommend that interested scholars extend their work to the Ulam stability of a fractional delay integro-differential equation with a Caputo derivative.

Author Contributions: Conceptualization, S.W.; writing-original draft preparation, S.W.; writing-review and editing, S.W. and F.M. All authors have read and agreed to the published version of the manuscript.

Funding: This research received no external funding.

Acknowledgments: The authors thank the reviewers for their helpful suggestions and comments.

Conflicts of Interest: The authors declare that they have no conflict of interest.

\section{References}

1. Ulam, S.M. A Collection of the Mathematical Problems; Interscience: New York, NY, USA, 1960.

2. Hyers, D.H. On the stability of the linear functional equation. Proc. Natl. Acad. Sci. USA 1941, 27, 222-224. [CrossRef]

3. Rassias, T.M. On the stability of the linear mapping in banach spaces. Proc. Am. Math. Soc. 1978, 72, 297-300. [CrossRef]

4. Aoki, T. On the stability of the linear transformation in banach spaces. J. Math. Soc. Jpn. 1950, 2, 64-66. [CrossRef]

5. Wang J.; Lv, L.; Zhou, Y. Ulam stability and data dependence for fractional differential equations with caputo derivative. Electron. J. Qual. Theory Differ. Equ. 2011, 63, 1-10. [CrossRef]

6. $\quad$ Liu, K.; Wang, J.; Zhou, Y.; O'Regan D. Hyers-ulam stability and existence of solutions for fractional differential equations with mittag-leffler kernel. Chaos Solitons Fractals 2020, 132, 1-8. [CrossRef]

7. Liu, K.; Feckan, M.; Wang, J. A fixed-point approach to the hyers-ulam stability of caputo-fabrizio fractional differential equations. Mathematics 2020, 8, 647. [CrossRef]

8. Jung, S.; Brzdek, J. Hyers-ulam stability of the delay equation $y(t)=y(t-\tau)$. Abstr. Appl. Anal. 2010, 13, 10. [CrossRef]

9. Zada, A.; Faisal, S.; Li, Y. On the Hyers-Ulam Stability of First-Order Impulsive Delay Differential Equations. J. Funct. Spaces Appl. 2016, 15, 1-6. [CrossRef]

10. Zada, A.; Shah, S.O. Hyers-Ulam Stability of First-Order Non-Linear Delay Differential Equations with Fractional Integrable Impulses. Hacet. J. Math. Stat. 2018, 47, 1196-1205. [CrossRef]

11. Luo, D.; Luo, Z. Existence and Hyers-Ulam stability results for a class of fractional order delay differential equations with non-instantaneous impulses. Math. Slovaca 2020, 70, 1231-1248. [CrossRef]

12. Kendre, S.D.; Unhale, S.I. On existence, uniqueness and Ulam's stability results for boundary value problems of fractional iterative integrodifferential equations. J. Appl. Math. Comput. 2020, 64, 503-517. [CrossRef]

13. Kucche, K.D.; Shikhare, P. Ulam Stabilities for Nonlinear Volterra Delay Integro-differential Equations. J. Contemp. Math. Anal. 2019, 54, 276-287. [CrossRef]

14. Huan, D.D.; Gao, H. A note on the existence of stochastic integro-differential equations with memory. Math. Methods Appl. Sci. 2015, 38, 1-15. [CrossRef]

15. Mokkedem, F.Z.; Fu, X. Approximate controllability of semi-linear neutral integro-differential systems with finite delay. Appl. Math. Comput. 2014, 242, 202-215. [CrossRef]

16. Desch, W.; Grimmer, R.; Schappacher, W. Wellposedness and wave propagation for a class of integrodifferential equations in Banach space. J. Differ. Equ. 1988, 74, 391-411. [CrossRef]

17. Gurtin, M.E.; Pipkin, A.C. A general theory of heat conduction with finite wave speed. Arch. Ration. Mech. Anal. 1968, 31, 113-126. [CrossRef]

18. Fujita, K. Integrodifferential equation which interpolates the heat equation and the wave equation. Osaka J. Math. 1997, 27, 309-321.

19. Berinde, V. Existence and approximation of solutions of some first order iterative differential equations. Miskolc Math. Notes 2010, 11, 13-26. [CrossRef]

20. Mi, T.; Stepan, G.; Takacs, D.; Chen, N. Vehicle shimmy modeling with pacejka's magic formula and the delayed tire model. J. Comput. Nonlinear Dyn. 2020, 15, 031005. [CrossRef]

21. He, Z.; Ni, D.; Zheng, M. Ergodicity and optimal control of a size-structured population model with delay. J. Syst. Sci. Math. Sci. 2018, 38, 1-15. [CrossRef] 
22. Wang, J.; Wei, W. A class of nonlocal impulsive problems for integrodifferential equations in Banach spaces. Results Math. 2010, 58, 379-397. [CrossRef]

23. Otrocol, D.; Ilea, V. Ulam stability for a delay differential equation. Cent. Eur. J. Math. 2013, 11, 1296-1303. [CrossRef]

24. Kendre, S.D.; Kharat, V.V.; Narute, R. On existence of solution for mixed iterative integrodifferential equations. Adv. Differ. Equ. Control Process. 2015, 15, 53-66. [CrossRef]

25. Sevgin, S.; Sevli, H. Stability of a nonlinear Volterra integro-differential equation via a fixed point approach. J. Nonlinear Sci. Appl. 2016, 9, 200-207. [CrossRef]

26. Kishor, D.K.; Pallavi, U.S. Ulam-Hyers Stability of Integrodifferential Equations in Banach Spaces via Pachpatte's Inequality. Asian-Eur. J. Math. 2018, 11, 1-19. [CrossRef]

27. Zada, A.; Ali, W.; Park, C. Ulam' type stability of higher order nonlinear delay differential equations via integral inequality of Grönwall-Bellman-Bihari's type. Appl. Math. Comput. 2019, 350, 60-65. [CrossRef]

28. Folland, G.B. Advanced Calculus; Prentice Hall: Indianapolis, IN, USA, 2001.

29. Pachpatte, B.G. Inequalities for Differential and Integral Equations; Academic Press: New York, NY, USA, 1998. [CrossRef]

30. Yuan, Z.; Yuan, X.; Meng, F.; Zhang, H. Some new delay integral inequalities and their appplications. Appl. Math. Comput. 2009, 208, 231-237. [CrossRef] 